\title{
Leishmaniose visceral entre índios no Estado de Roraima, Brasil. Aspectos clínicoepidemiológicos de casos observados no período de 1989 a 1993
}

\author{
Visceral leishmaniasis among Indians of the State of Roraima, Brazil. Clinical and \\ epidemiologic aspects of the cases observed from 1989 to 1993
}

\author{
Jorge Augusto 0. Guerra ${ }^{1}$, Marcus Luíz B. Barros ${ }^{1,2}$, Nelson Ferreira Fé1, Marcus Vinitius F. \\ Guerra $^{1,2}$, Eloy Castellon ${ }^{3}$, Marcilene Gomes Paes ${ }^{1}$ e Ítalo A. Sherlock ${ }^{4}$
}

\begin{abstract}
RESUM0
Descreve-se o perfil epidemiológico da leishmaniose visceral entre índios no Estado de Roraima, Brasil, baseado na ocorrência de casos humanos observados e nos inquéritos caninos e entomológicos realizados no período de 1989 a 1993. Foram registrados 82 casos humanos de leishmaniose visceral em seis dos oito municípios então existentes no estado; houve predomínio de 69,5\% para o sexo masculino entre os casos observados. A maioria (52,4\%) dos casos foi entre crianças de zero a dez anos de idade. Registrou-se o índice de 10,3\% de infecção canina natural, entre 3.773 cães examinados em 74 localidades pesquisadas. A Lutzomyia longipalpis foi encontrada nas áreas de maior prevalência da doença, em 31 localidades diferentes. Os casos humanos, caninos e vetores estão concentrados em regiões onde predominam serras e lavrados, áreas características de ocorrência da leishmaniose visceral americana. A introdução e intensificação das atividades garimpeiras na região podem ter contribuído para a introdução da doença.
\end{abstract}

Palavras-chaves: Leishmaniose visceral. Perfil epidemiológico. Índios. Estado de Roraima. Brasil.

\begin{abstract}
A description of the epidemiological profile of visceral leishmaniasis among Indians in the State of Roraima, Brazil, was based on the clinical characteristics of human and dog disease, ecological aspects of the area where the cases occurred and entomologic investigations performed from 1989 to 1993. The 82 human cases were reported in six out of eight Counties that existed then in the State; there was a $69.5 \%$ predominance of male cases among those registered and a greater (52.4\%) occurrence of the disease in children from zero to ten years old. The rate of natural infection was $10.3 \%$ out of 3,773 dogs examined in 74 different locations. Lutzomyia longipalpis was found in 31 areas with greater prevalence of the disease. The human and animal cases as well as the vectors were concentrated in areas where mountains and arable soil predominate, typical locations for the occurrence of American visceral leishmaniasis.
\end{abstract}

Key-words: Visceral leishmaniasis. Epidemiologic profile. Indians. Roraima State. Brazil.

0 Estado de Roraima possui na sua região central campos naturais ou savanas, conhecidos localmente como lavrado, e florestas tropicais ao sul ${ }^{4}$. Ao norte, existe um complexo de montanhas, em cujos vales, pelo lado brasileiro, foram detectados, pela primeira vez no estado, em 1989, os primeiros casos autóctones de leishmaniose visceral ${ }^{1}{ }^{20} \mathrm{e} \mathrm{a}$ presença da Lutzomya longipalpis ${ }^{28}$. Antes desse período não havia registro de casos de leishmaniose visceral em Roraima.

0 estado abriga diversas nações indígenas, destacandose entre elas os Macuxi, já mesclados aos demais grupos

1. Fundação de Medicina Tropical do Amazona, Manaus, AM. 2. Universidade Federal do Amazonas, Manaus, AM. 3 Instituto Nacional de Pesquisas da Amazônia, Manaus, AM. 4. Fundação Oswaldo Cruz, Salvador, BA.

Parte da disseração de mestrado apresentado no programa de pós-graduação da FIOCRUZ em convênio com o IMTM.

Endereço para correspondência: Dr. Jorge Augusto de Oliveira Guerra. Gerência de Leishmanioses/FMTAM. Av. Pedro Teixeira 25, D. Pedro I, 69040-000 Manaus, AM.

Fax: 5592 238-3762

e-mail: jguerra@ horizon.com.br

Recebido para publicação em 8/8/2003

Aceito em 22/5/2004 
étnicos e raciais e compondo a maioria dos habitantes de Roraima e cujo território oficialmente demarcado está no norte, mas que na verdade, se distribuem por praticamente todo 0 estado; e a Nação Yanomami, situada a noroeste (Figura 1). Estes índios têm tido suas terras freqüentemente invadidas por garimpeiros ${ }^{30} 0$ que tem gerado grandes conflitos sociais ${ }^{26}$.

№ final do ano de 1988 foi enviada ao Serviço de Hematologia do Amazonas (HEMOAM) uma criança Macuxi, procedente do Município de Normandia RR, com suspeita clínica de leucose. Apresentava quadro febril, emagrecimento e hepato-esplenomegalia. No material obtido por punção biópsia de medula óssea ( PMO) , os profissionais daquela instituição e do Instituto de Medicina Tropical de Manaus ( IMTM) constataram a presença de formas amastigotas de Leishmania no esfregaço realizado, identificando-se assim 0 primeiro caso autóctone de LV do Estado de Roraima ${ }^{20}$.

A partir de 1989 houve importante aumento do número de casos, notificados em diversos municípios ${ }^{526}$. As tentativas de se estabelecer um plano de controle da endemia eram exercidas mediante ações da Fundação Nacional de Saúde de Roraima (FNS/RR), associada a órgãos de pesquisa, como 0 Instituto de Medicina Tropical de Manaus (IMTM) e Instituto Nacional de Pesquisas da Amazônia (INPA).

Com 0 objetivo de descrever esse novo foco, e de traçar 0 perfil clínico epidemiológico da endemia no Estado de Roraima, realizamos um estudo dos casos humanos atendidos nos hospitais dessa área, dos inquéritos caninos e dos vetores.

\section{MATERIAL E MÉTODOS}

Este trabalho descreve os casos detectados e registrados entre os anos de 1989 e 1993, em dois momentos às vezes simultãneos: um estudo retrospectivo, cujas informações foram coletadas nas instituições que atendiam os doentes, notificavam aos casos e realizavam diagnóstico e pesquisa dos mesmos - a Casa do Índio de Roraima (CIR) , o Hospital Coronel Mota (HCM) , a Fundação Nacional de Saúde de Roraima (FNS/RR), o Laboratório Central de Saúde ( LACEN) e 0 (IMTM), constituíram-se nas instituições envolvidas no estudo. Vale salientar que a maior parte das informações utilizadas neste trabalho foi coletada pelos autores, que trabalharam nas atividades de campo.

Em uma segunda etapa foram realizados trabalhos de campo no período compreendido entre 1992 e 1993, constituindo-se em um estudo prospectivo.

Os critérios utilizados no diagnóstico dos casos humanos foram baseados nos aspectos clínicos, epidemiológicos e laboratoriais, que incluíam: punção biópsia de medula óssea, punção esplênica e sorologia pela reação de imunofluorescência indireta (RIFI). Para os inquéritos caninos foram considerados diagnósticos: a sorologia positiva (RIFI), em qualquer titulação, e/ou o encontro de parasitas em material coletado de baço ou fígado de cães sacrificados; nos inquéritos entomológicos considerou-se a presença da Lu longipalpis, coletada em armadilhas CDC.

A análise estatística das informações foi realizada através do programa EPI info versão 6.0 (CDC) . Na análise da prevalência foi utilizado 0 teste do qui-quadrado, considerando 0 nível de significância de $5 \%(p \leq 0,05)$.

\section{RESULTAD0S}

0 maior número de casos foi registrado em 1991 com 26 (31,7\%) ocorrências ( Tabela 1) ; e o menor número em 1989 com $10(12,2 \%)$ casos.

Em seis municípios, dentre os oito existentes à época no Estado de Roraima, registraram-se 82 casos de LV. A maior

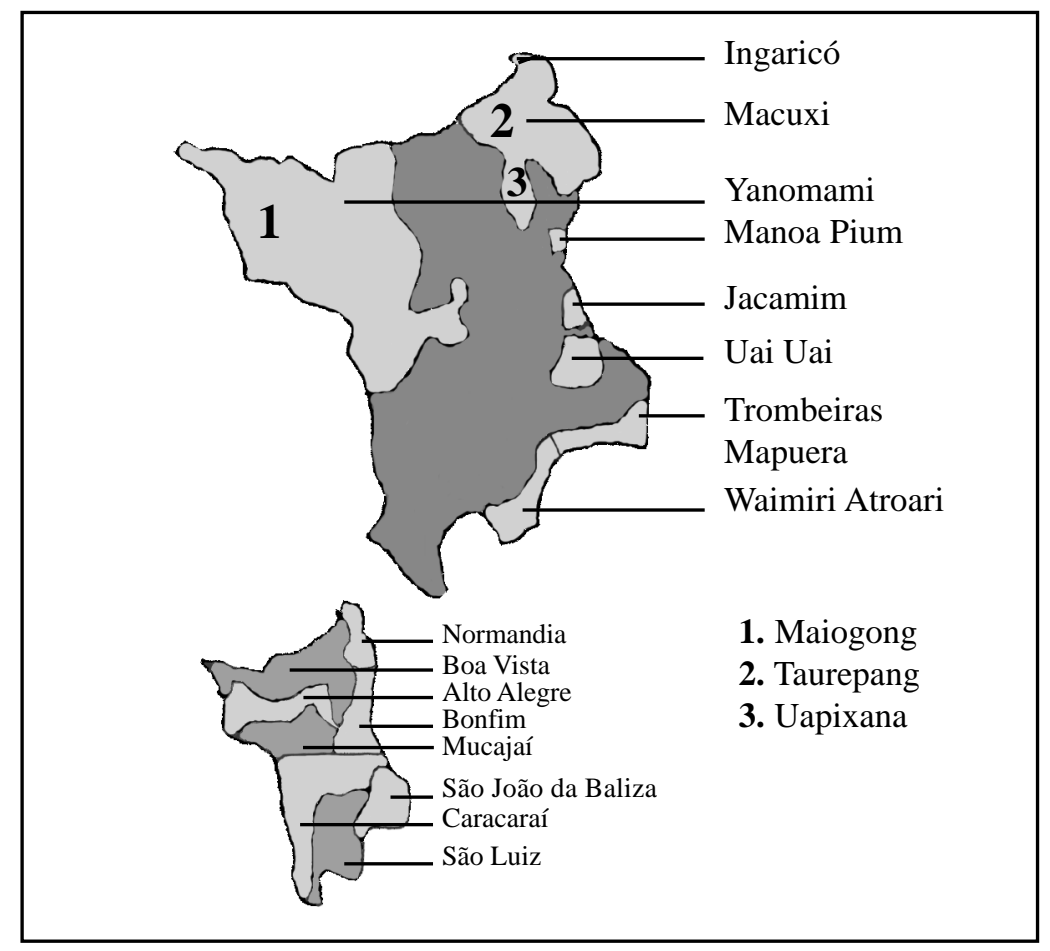

Figura 1 - Divisão política e principais áreas indígenas de Roraima. 
Tabela 1 - Leishmaniose visceral entre índios no Estado de Roraima, Brasil - 1989 a 1993. Distribuição anual dos casos humanos.

\begin{tabular}{lcrc}
\hline Ano & $N^{p}$ casos & Percentagem & Incidência por ano(x 10.000) \\
\hline 1989 & 10 & 12,2 & 0,45 \\
1990 & 15 & 18,3 & 0,69 \\
1991 & 26 & 31,7 & 1,19 \\
1992 & 20 & 24,4 & 0,91 \\
1993 & 11 & 13,4 & 0,91 \\
\hline Total & 82 & 100,0 & 0,83 \\
\hline
\end{tabular}

ocorrência foi em Normandia com 36 (44\%) casos, seguido de Boa Vista com 25 (30,5\%), Alto Alegre com 10 (12,2\%) e Mucajaí com 4 (4,9\%). São João da Baliza e Bonfim apresentaram 0 menor número de casos, 1 (1,2\%) para cada município. Três outros pacientes foram diagnosticados em Roraima, mas provavelmente adquiriram a doença do outro lado da fronteira, na República da Guiana, pois eram garimpeiros procedentes daquele país (Tabela 2).

Tabela 2 - Leishmaniose visceral entreíndios no Estado de Roraima, Brasil 1989 a 1993. Distribuição dos casos humanos por município de ocorrência.

\begin{tabular}{lrr}
\hline Município & $N^{0}$ casos & Percentagem \\
\hline Normandia & 36 & 44,0 \\
Boa Vista & 25 & 30,5 \\
Alto Alegre & 10 & 12,2 \\
Mucajá & 4 & 4,9 \\
São João da Baliza & 1 & 1,2 \\
Bonfim & 1 & 1,2 \\
Guiana & 3 & 3,6 \\
Sem registro & 2 & 2,4 \\
\hline Total & 82 & 100,0 \\
\hline
\end{tabular}

Na distribuição dos casos humanos, observou-se o predomínio entre comunidades indígenas, com $71(86,6 \%)$ casos, a maioria pertencente à Nação Macuxi 55 (67,1\%), seguidos dos Yanomami com $14(17,1 \%)$ casos e o menor número entre os índios Ingaricó com 2 (2,4\%). Em onze prontuários não havia registros de etnia; sabe-se apenas que eram garimpeiros, procedentes de diversas localidades, dentro e fora do Estado de Roraima.

0 sexo masculino apresentou 57 (69,5\%) casos e o feminino 25 (30,5\%) (Tabela 3). Aanálise estatística mostrou significância para 0 sexo masculino em $69,5 \%$ dos casos $(p<0,0001)$.

A idade variou entre 1 e 65 anos, sendo a média global da idade de 8,7 anos. A faixa etária de maior ocorrência estava entre 0 e 10 anos, com $43(52,43 \%)$ casos (Tabela 3), sendo que 33 pacientes possuíam até cinco anos de idade. Apenas um

Tabela 3 - Leishmaniose visceral entreíndios no Estado de Roraima, Brasil - 1989 a 1993. Distribuição dos casos humanos por idade/sexo.

\begin{tabular}{lrrrrr}
\hline & \multicolumn{2}{c}{ Sexo } & & \multicolumn{2}{c}{ Casos } \\
\cline { 2 - 3 } \cline { 5 - 6 } Idade & masc & fem & & $\mathrm{n}^{0}$ & $\%$ \\
\hline 0 a 10 & 21 & 22 & & 43 & 52,4 \\
11 a 20 & 10 & 2 & 13 & 15,9 \\
21 a 30 & 11 & 0 & 11 & 13,4 \\
31 a 40 & 6 & 0 & 6 & 7,3 \\
41 a 50 & 8 & 0 & 8 & 9,8 \\
$>50$ & 0 & 1 & 1 & 1,2 \\
\hline Total & 57 & 25 & 82 & 100,0 \\
\hline
\end{tabular}

paciente possuía idade acima de 50 anos. A análise estatística mostrou significância para a faixa etária compreendida entre zero a dez anos, observada em $52,4 \%$ dos casos ( $p<0,0001$ ).

As manifestações clínicas predominantes foram febre, anemia, diarréia e hepatoesplenomegalia (Tabela 4).

Tabela 4 - Leishmaniose visceral entreíndios no Estado de Roraima, Brasil - 1989 a 1993. Sinais e sintomas apresentados entre os casos humanos

\begin{tabular}{lrr}
\hline Sintomas & $\mathrm{N}^{0}$ casos & Percentagem \\
\hline Febre & 72 & 87,8 \\
Anemia & 42 & 51,2 \\
Diarréia & 36 & 43,9 \\
Hepatoesplenomegalia & 29 & 35,4 \\
Hemorragias & 17 & 20,7 \\
Emagrecimento & 14 & 17,1 \\
Vômitos & 13 & 15,8 \\
Edema & 12 & 14,6 \\
Dispnéia & 8 & 9,7 \\
Dor torácica & 7 & 8,5 \\
Outros & 18 & 21,4 \\
\hline
\end{tabular}

Entre as doenças associadas, as parasitoses intestinais predominaram, com $28(34,1 \%)$ casos, sendo a maioria ancilostomíase 13 (46,42\%), seguida de ascaridíase e amebíase em nove, e giardíase em seis doentes. Amalária esteve associada a $22(26,8 \%)$ casos, sendo onze por Plasmodium vivax, oito por P. falciparum e três por malária mista. Seis pacientes eram portadores de tuberculose pulmonar ( Tabela 5).

Tabela 5 - Leishmaniose visceral entreíndios no Estado de Roraima, Brasil - 1989 a 1993. Distribuiç̧ão dos casos humanos por patologias associadas

\begin{tabular}{llr}
\hline Patologias & $N^{j}$ casos & Percentagem \\
\hline Parasitoses intestinais & 28 & 34,1 \\
Malária & 22 & 26,8 \\
Pneumopatias & 17 & 20,7 \\
Doenças cutâneas & 9 & 11,0 \\
Gastrenterite & 4 & 4,9 \\
Otite & 2 & 2,4 \\
Outras & 5 & 6,1 \\
\hline
\end{tabular}

0 diagnóstico de LV foi confirmado através da punção biópsia de medula óssea (PM0), em 50 (61\%) casos, da punção esplênica em 11 (13,4\%) e da sorologia em 8 (9,8\%). Em alguns pacientes foi utilizado mais de um método. Em 13 (15,8\%) casos, foi utilizado o critério clínico-epidemiológico pois não havia registros de confirmação laboratorial; apenas 0 indicativo de registro e tratamento para LV (Tabela 6).

Após isolamento em culturas, a espécie de Leishmania desses casos humanos de Roraima foi identificada no Instituto Evandro Chagas (Belém-PA), através de isoenzimas, como Leishmania (Leishmania) chagasi.

Tabela 6 - Leishmaniose visceral entreíndios no Estado de Roraima, Brasil - 1989 a 1993. Critérios diagnósticos utilizados nos casos humanos.

\begin{tabular}{llr}
\hline Método utilizado & № casos & Percentagem \\
\hline Punção de medula óssea & 50 & 61,0 \\
Clínico-epidemiológico & 13 & 15,8 \\
Punção esplênica & 11 & 13,4 \\
Sorológico & 8 & 9,8 \\
\hline Total & 82 & 100,0 \\
\hline
\end{tabular}


Em 65 (79,3\%) doentes examinados a média geral do número de hemácias foi de $2.933 .230 / \mathrm{mm}^{3}$; a de hemoglobina foi de $8,83 \mathrm{~g} / \mathrm{dl}$.

Em 68 doentes examinados a média de leucócitos foi de $3.900 / \mathrm{mm}^{3}$.

№ inquérito canino foram examinados 3.773 cães em todo 0 estado, distribuídos entre 74 malocas, garimpos, fazendas, vilas, sítios e missões. Foram observados 10,4\% (390 casos) de positividade pela RIFI. Na distribuição dos casos caninos por municípios, São Luís apresentou maior percentual de positividade, com 16,7\% de sorologias positivas (três amostras positivas em 18 cães examinados). Entretanto, analisando valores absolutos, observaram-se 254 (10,8\%) amostras positivas em Normandia e 102 (13,2\%) em Boa Vista. Nos municípios de Mucajaí e Caracaraí,

Tabela 7 - Leishmaniose visceral no Estado de Roraima, Brasil - 1989 1993. Inquérito canino. Distribuição de casos positivos por Município.

\begin{tabular}{lrrr}
\hline Município & Examinados & Positivos & Percentagem \\
\hline Normandia & 2.335 & 254 & 10,8 \\
Boa Vista & 869 & 102 & 13,2 \\
Alto Alegre & 316 & 9 & 2,9 \\
Bonfim & 174 & 22 & 12,6 \\
São Luís & 18 & 3 & 16,7 \\
Mucajá & 34 & 0 & 0 \\
Caracaraí & 27 & 0 & 0 \\
\hline Total & 3.773 & 390 & 10,3 \\
\hline
\end{tabular}

Tabela 8 - Leishmaniose visceral no Estado de Roraima, Brasil. Número de espécimes de Lutzomyia longipalpis coletados no estado de 1989 a 1992.

\begin{tabular}{lrr}
\hline Ano & \multicolumn{2}{c}{ Espécimes coletados } \\
\cline { 2 - 3 } & \multicolumn{1}{c}{$\mathrm{n}^{0}$} & $\%$ \\
\hline 1989 & 540 & 6,5 \\
1990 & 88 & 1,1 \\
1991 & 470 & 5,6 \\
1992 & 7.242 & 86,8 \\
\hline Total & 8.340 & 100,0 \\
\hline
\end{tabular}

não havia registros de cães com sorologia positiva para leishmaniose ou de quadro clínico compatível com IV canina. Năo foram realizados inquéritos caninos em São João da Balisa (Tabela 7).

Em apenas dois municípios do estado foi registrada a presença da Lu. longipalpis: Normandia, em 26 localidades e Boa Vista em 5 (Figura 2).

No ano de 1992 foi observado o maior número de Lu. longipalpis, com 7.246 (86,8\%) espécimes capturadas. 0 menor número correspondeu ao ano de 1990, com 88 (1,1\%) (Tabela 8).

\section{DISCUSSÃ0}

De acordo com os dados do Ministério da Saúde, Fundação Nacional de Saúde ${ }^{6}{ }^{21}$, foram registrados oficialmente 153 casos de calazar em Roraima, no período de 1989 a 1993, com a

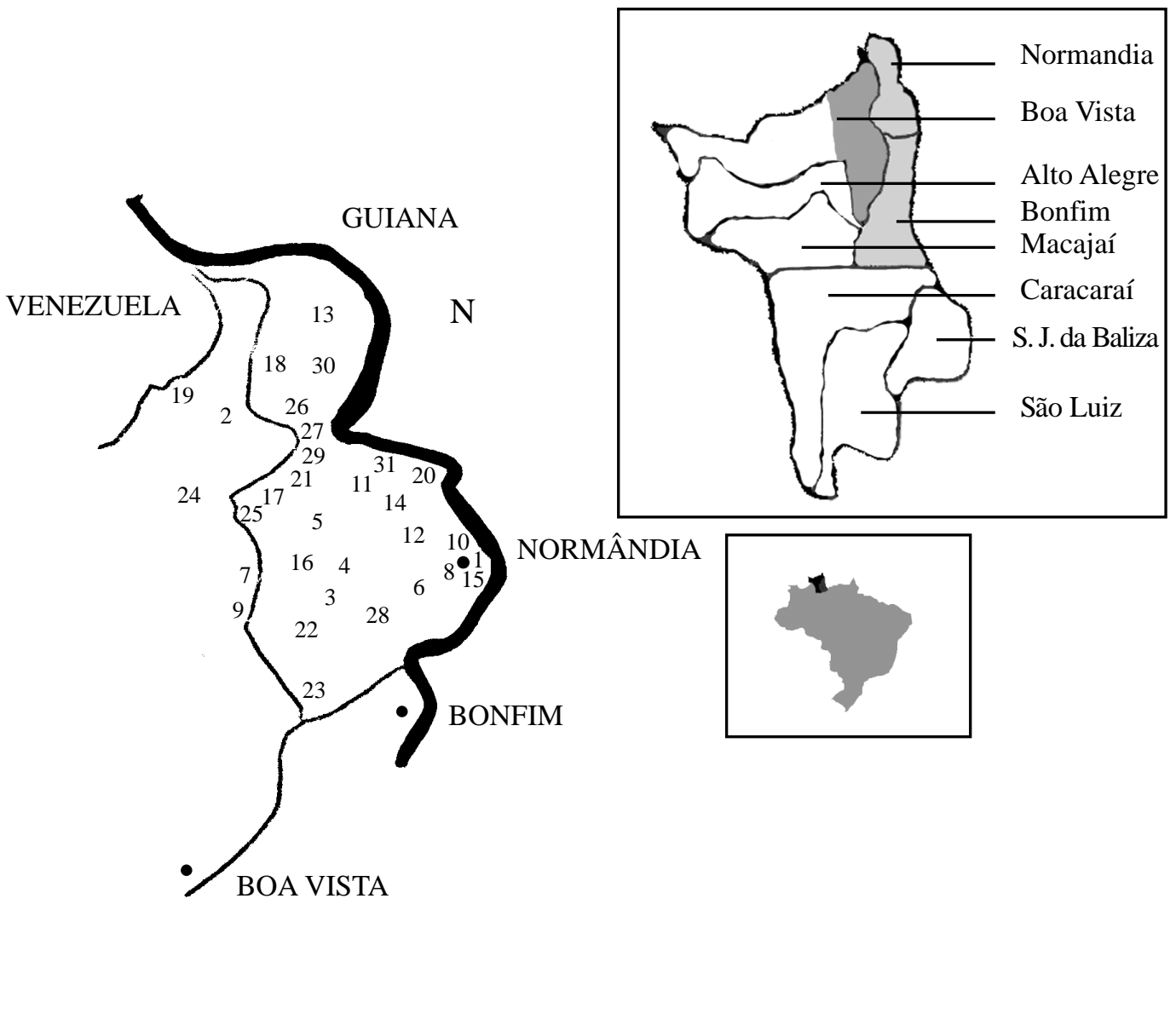

Figura 2 - Localidades em Roraima onde foram realizadas coletas de espécimes de Lutzomyia longipalpis - 1989 a 1992. 
seguinte distribuição: cinco casos em 1989, seis em 1990, 41 em 1991, 62 em 1992 e 39 em 1993. Há um desacordo entre o número de casos oficialmente registrados e aqueles encontrados em nosso estudo, refletindo uma subnotificação nos dois primeiros anos. Como se observa, esses dados mostram um aumento inicial progressivo no número de casos, o que provavelmente foi fruto da busca ativa exercida pelos órgãos de pesquisa associados à FNS. Posteriormente, houve um decréscimo no número de casos, devido às dificuldades em manter-se, nos anos seguintes, uma ação continuada por parte desses órgãos oficiais. De 1994 a 2000, a média de casos foi de 18,716 ${ }^{16}$.

Essa situação traduza dificuldade e conseqüente descontinuidade das ações exercidas para a busca, diagnóstico e notificação dos casos de IV. 0 órgão que melhor notifica a existência de casos é a Casa do Índio, pelo interesse por parte do Distrito Sanitário Yanomami (DSY) em registrar doenças ocorrentes entre os indígenas ${ }^{26}{ }^{30}$. Todavia há falhas nas informações básicas, como a procedência, sexo, idade e data do início dos sintomas, em face das dificuldades culturais de alguns grupos.

Nossos resultados indicam que os municípios de Normandia e Boa Vista, cujo relevo de lavrado e serras correspondem ao padrão ecológico comum da leishmaniose visceral neo-tropical ${ }^{111} 1819233334$, abrigam maior número de casos. Entretanto, a doença também ocorre em áreas de transição de floresta e lavrado, relacionada a fatores como 0 caráter migratório dos indígenas, que fazem deslocamentos freqüentes para fora de suas reservas. Essa característica é observada entre os Macuxi, grupo predominante dentro dasáreas de ocorrência de LV, e entre os Yanomami e os Ingaricó.

Destacam-se ainda, como fatores intervenientes, a introdução e a posterior intensificação da atividade garimpeira naquelas áreas, fenômeno que proporcionou uma grande imigração de nordestinos, muitos provenientes de áreas endêmicas de calazarr ${ }^{31}$.

A despeito dos primeiros registros haverem ocorrido entre os Macuxis, percebe-se num segundo momento, casos sendo diagnosticados entre os Yanomami, e em Centros de atenção à saúde em Boa Vista e até mesmo em Manaus.

0 que se observa é que a epidemia do calazar ocorre predominantemente entre populações indígenas da região, mas foram provavelmente os garimpeiros, em sua maioria provenientes de áreas de reconhecida endemia, que a introduziram em Roraima, cuja ecologia favorece a ocorrência do calazar, favorecendo, desta forma, a produção de casos autóctones.

Apesar de a epidemia ser extensiva à Guyana, onde também ocorre atividade garimpeira, não há registros oficiais de casos naquele país até 0 período deste estudo ${ }^{37}$.

Pode-se aventar a hipótese de que a localização geográfica da área, em proximidade da transição com 0 lavrado, por motivos ainda não esclarecidos, propicie um aumento no potencial de transmissão da doença ${ }^{26}$. Costa et al ${ }^{11}$ alertam para a necessidade de estudos da ecologia nessas áreas de transição entre florestas, cerrados e serras.

Ocorre predomínio de casos no sexo masculino, porém na faixa etária de zero a dez anos há tendência à igualdade para ambos os sexos, sendo justamente nessa faixa etária que se observa a maior casuística, 0 que é típico da LV nas
Américas $^{14} 15$ 37. Foi observado ainda que a Leishmania isolada dos casos humanos de Roraima foi caracterizada como Leishmania (L) chagasi ${ }^{29}$, cujo perfil epidemiológico de casos humanos tem essas características.

Os indígenas utilizam diversos alimentos provenientes de suas atividades de caça, pesca, coleta e agricultura; a abundância ou a carência desses recursos em determinada região é fator determinante na escolha de novos sítios de habitação. Sua grande mobilidade e dispersão espacial são estratégias de adaptação fundamentais.

Em certos locais, principalmente na área Yanomami, a disponibilidade de recursos alimentares foi muito diminuída durante a exploração garimpeira. Esse fato agravou ainda mais o quadro de morbimortalidade, ao desviar a mão de obra indígena para o garimpo. A introdução dos produtos de consumo trazidos pelos garimpeiros teve alta aceitação por parte dos nativos, criando dependências nessas populações. Tudo isso influenciou o grau de nutrição e consequentemente a ocorrência e evolução de doenças como a tuberculose e a própria IV, como salientado por alguns autores ${ }^{237}$.

Os índios Macuxi têm por costume visitar os parentes em longínquas comunidades, levando toda a família e seus animais, inclusive os cães, permanecendo muito tempo nesses locais. Esses movimentos migratórios funcionam como fator disseminador da doença. Os cães são muito valorizados na região, pois além do valor afetivo que lhes é atribuído, servem para a caça, ajudando na busca de alimentos e na subsistência do homem.

Entre as doenças associadas a IV, destacam-se a malária, a tuberculose (esta última, principalmente entre os Yanomami) e as parasitoses intestinais como fatores agravantes ${ }^{7}$, conforme tem sido observado em diversas comunidades indígenas. Associados à tuberculose, há registros de seis casos. 0 estado de nutrição dos Yanomami é muito precário, devendo contribuir para essa concomitância ${ }^{15}$.

A associação entre malária e LV parece freqüente. Entretanto, poucos trabalhos têm discutido sobre essa associação. Na Índia, foram descritos alguns desses $\operatorname{casos}^{27}$, comentando-se que os pacientes permanecem com quadros febris fazendo uso repetido de antimaláricos, sem melhora. Em Roraima, a grande maioria dos pacientes de IV procede de áreas de transmissão concomitante de malária e já apresentou episódios anteriores de malária ou utilizou antimaláricos, o que pode muitas vezes causar confusão para definir os aspectos clínicos evolutivos de cada uma das patologias.

0 registro de casos de IV canina foi feito em praticamente todo 0 Estado de Roraima. Em 91 localidades registraram-se cães com sorologia positiva, com ou sem sinais clínicos de doença. Houve um predomínio acentuado nos Municípios de Normandia e Boa Vista, ao norte do estado, coincidindo com a distribuição dos casos humanos e dos vetores. Nosso trabalho apresenta 3.773 cães examinados tendo sido observada uma soroprevalência de $10,3 \%$, pela RIFI. Coutinho et a ${ }^{12}$ encontraram no Rio de Janeiro taxas de 4,3\% entre cães procedentes de área de transmissão de LV, 8,6\% nos de procedência de transmissão de LTA e 12\% onde ambas as doenças ocorreram. Sherlock \& Almeida ${ }^{35}$ admitem, pela primeira vez, a existência de áreas de transmissão concomitante de leishmaniose visceral e tegumentar no Estado da Bahia. Senra et $a l^{33}$ examinaram 4.593 cães em Santarém, no Estado do Pará, e encontraram 32,3\% de soropositividade. 
0 Manual de Controle do Calazar do Ministério da Saúde enfoca que as ações no controle da doença devem ser dirigidas aos cães ${ }^{17}$. Além da prevalência da infecção nos cães ser bem maior que no homem, 0 cão abriga parasitos na pele e infecta mais facilmente 0 transmissor propiciando a infecção entre este e 0 homem $^{24}$. A soroprevalência canina encontrada nos municípios da Região Sul do estado pode ser explicada pela ocorrência da LTA. Costa et al ${ }^{10}$ advertem para o problema do uso da RIFI como indicador de prevalência de infecção canina, em áreas de transmissão concomitante com LTA.

A partir de 1991, houve uma intensificação nas atividades de controle, que incluíram diagnóstico e tratamento dos casos humanos e sacrifício dos cães doentes ou com sorologia positiva, pois a doença começava a expandir-se em Roraima, com 0 aparecimento de novos casos em áreas distantes do foco inicial. 0 ano de 1993 culmina com 1.467 cães examinados e percentuais de 15,9\% de infecção, abrangendo cinco municípios do estado, sendo Normandia, com 254 $(10,8 \%)$ cães infectados e Boa Vista, com $102(13,2 \%)$ os municípios com maior número de registros.

No Brasil, a ocorrência de Lu. longipalpis tem sido amplamente associada ao aparecimento de casos humanos ou caninos $^{25}$. De acordo com os dados da literatura ${ }^{13}{ }^{31}{ }^{36}$, os locais de maior ocorrência de Lu. longipalpis são os chamados boqueirões e pés de serras, onde abundam rochas calcárias. Na Amazônia, a presença da Lu. longipalpis foi registrada no Estado do Pará, associada à leishmaniose visceral, desde os primeiros casos descritos no País ${ }^{8}$, quando foi encontrado um grande número desse flebótomo no intra e peridomicílio humano, associado também à infecção canina. No Estado de Roraima, 0 vetor incriminado também foi a Lu. longipalpis ${ }^{28}$.

A Lu. longipalpis facilmente invade 0 peridomicílio e abrigos de animais domésticos, sendo capturada em galinheiros ${ }^{23} 32{ }^{37}$ e nos fornos de fazer farinha, que servem de abrigo a cães e galinhas ${ }^{22}{ }^{23}$. A Lu. longipalpis prefere picar esses animais ao homem, fato também verificado na Colômbia ${ }^{9}$.

A Lu. longipalpis foi o flebotomíneo mais freqüentemente encontrado em associação com a infecção canina e humana em Roraima. Durantenossas observações de campo, esse inseto mostrou bastante avidez em picar humanos, sendo encontrada uma grande densidade deles picando garimpeiros, dentro e fora de suas barracas, nos arredores das casas de farinha e dentro dos fornos.

Apesar de inquéritos entomológicos terem sido realizados na área Yanomami de Roraima, pelo INPAe pela FNS, durante o período deste estudo, não se capturaram flebotomíneos que pudessem ser incriminados como transmissores de LV naquela área.

Não há registros da ocorrência da Lu. longipalpis no Estado de Roraima antes de 1989.

Observa-se, portanto, que a introdução do calazar em Roraima é recente. Há necessidade de novos estudos, dentro de uma visão antropológica, direcionados principalmente a essas populações, predominantemente indígenas, além da manutenção de medidas que incluem vigilância epidemiológica para casos caninos e humanos, além de vigilância entomológica, visando manter 0 controle da endemia.

\section{AGRADECIMENTOS}

A Omarina Andrade Guerra e Alcidéa Rego Bentes e Sousa pela revisão e sugestões do manuscrito e a Gustavo Henrique Johanson pela revisão do abstract. À FNS/RR pelo apoio logístico.

\section{REFERÊNCIAS BIBLIOGRÁFICAS}

1. Araújo Filho NA, Guerra MVF, Passos LNM, Paes MG, Barros MLB, Guerra ALS, Santos DM, Carvalho MPS, Sena CAR. Calazar no Estado de Roraima, Brasil In: Resumos do XI Congresso Brasileiro de Parasitologia, Rio de Janeiro p.16, 1989.

2. Badaró R, Jones TC, Carvalho EM, Sampaio D, Reed SG, Barral A, Texeira R, Johnson Jr. WD.. New perspectives on a subclinical form of Visceral Leishmaniasis. Journal of Infectious Diseases 154:1003-1012, 1986.

3. Badaró R, Rocha H, Carvalho EM, Queiroz AC. Leishmania donovani: An opportunistic microbe associated with progressive disease in three immunocompromised patients. The Lancet 22:467-469, 1986.

4. Boher CBA, Gonçalves LMC. Vegetação. In: Geografia do Brasil - Região Norte. Fundação Instituto Brasileiro de Geografia e Estatística, Rio de Janeiro, RJ, p. 137-168, 1991.

5. Castellon EB, Domingos E. On the focus of Kala-azar in the state of Roraima, Brazil. Memórias do Instituto Oswaldo Cruz 86: 375, 1991.

6. CENEPI. Ministério da Saúde. Fundação Nacional de Saúde. Centro Nacional de Epidemiologia 6, p.127, 1993.

7. Cerf BJ, Jones TC, Badaró R, Sampaio D, Teixeira R, Johnson Jr. WD. Malnutrition as a risk factor for severe visceral leishmaniasis. Journal of Infectious Diseases 156:1030-1033, 1987.

8. Chagas E, Cunha AM, Castro GO, Ferreira LO, Deane L, Deane G, Guimarães FN, Von Paungartem M, Sá B. Leishmaniose visceral americana (Relatório dos trabalhos realizados pela comissão encarregada do estudo da leishmaniose visceral americana em 1937). Memórias do Instituto Oswaldo Cruz 33:89-283, 1938.

9. Corredor A, Gallego JF, Tesh RB, Morales A, Carrasquilla CF, Young DG, Kreutzer RD, Boshell J, Palau MT, Caceres E, Pelaez D. Epidemiology of visceral leishmaniasis in Colombia. American Journal of Tropical Medicine and Hygiene 40:480-486, 1989.

10. Costa CA, Genaro O, Lana M, Magalhães PAM, Michalick MSM, Melo MN, Costa RT, Magalhães NM, Mayrink W. Leishmaniose visceral canina: avaliação da metodologia utilizada em inquéritos epidemiológicos Revista da Sociedade Brasileira de Medicina Tropical 24:21-25, 1991.

11. Costa $\mathrm{CH}$, Pereira HF, Araújo MV. Epidemia de leishmaniose visceral no Estado do Piauí, Brasil, 1980-1986. Revista de Saúde Pública de São Paulo 24:361-372, 1990

12. Coutinho SG, Nunes MP, Marzochi MCA, Tramontano NA. A survey for american cutaneous and visceral leishmaniasis among 1,342 dogs from areas in Rio de Janeiro (Brazil) where the diseases occur. Memórias do Instituto Oswaldo Cruz 80:17-22, 1985.

13. Deane, LM. Leishmaniose Visceral no Brasil. Estudos sobre reservatórios e transmissores realizados no Estado do Ceará. Serviço Nacional de Educação Sanitária. Rio de Janeiro, Brasil. 162p. 1956.

14. Evans G, Teixeira MJ, Mcauliffe IABV, Vasconcelos AW, Sousa AQ, Lima JW, Pearson RD. Epidemiology of Visceral Leishmaniasis in Northeast Brazil. Journal of Infectious Diseases 166:1124-1132, 1992.

15. Fundação Nacional de Saúde. Centro Nacional de Epidemiologia. Programa Nacional de controle das leishmanioses. Manual de controle do Calazar, 1994.

16. Fundação Nacional de Saúde. http://www.funasa.gov.br/guia epi/imagens/ serie_historica_1980_2001/leishm_visceral_uf.pdf, 2001.

17. Fundação Nacional de Saúde. Política Nacional de Atenção à Saúde dos Povos Indígenas 2ª Edição, Ministério da Saúde, 2002.

18. Gerônimo SO, Mackay S, Costa RM, Sweet J, Nascimento ET, Luz KG, Fernandes MZ, Jeningan J, Pearson RD. An urban outbreak of visceral 
leishmaniasis in Natal, Brazil. Transactions of the Royal Society of Tropical Medicine and Hygiene 88:386-388, 1993.

19. Guerra JAO, Fé NF, Rodrigues C, Silva C, Barros MLB, Guerra MVF. Leishmaniose visceral no Estado de Roraima: casos registrados no período de 1989 a 1994. Revista da Sociedade Brasileira de Medicina Tropical. 29( supl. I) :142. 1995.

20. Guerra MVF, Araújo Filho NA, Paes MG, Barros MLB, Sá RC, Ramos ED. Aspectos clínicos do calazar no Estado de Roraima, Brasil In: Resumos do XI Congresso Brasileiro de Parasitologia, Rio de Janeiro p.17, 1989.

21. Lacerda MM. The Brazilian leishmaniasis control program. In: Brandão Filho S (eds) Research and control of leishmaniasis in Brazil. Proceedings of a National Workshop. Recife, pp.287-299, 1993.

22. Lainson R. The American leishmaniasis: some observations on their ecology and epidemiology. Transactions of the Royal Society of Tropical Medicine and Hygiene 77:569-596, 1983.

23. Lainson R, Shaw JJ, Silveira FT, Braga RR, Ryan L, Póvoa MM, Ishikava EAY. A Leishmania e as leishmanioses. In: Instituto Evandro Chagas ( org) 50 anos de contribuição às ciências biológicas e a medicina tropical. Fundação Serviços de Saúde Pública e Editora Globo, Ministério da Saúde, Belém, 2 vols, p. 1986.

24. Marzochi MCA, Coutinho SG, Sabrosa PC, Sousa MA, Sousa PP, Toledo LM, Filho FBR. Leishmaniose visceral canina no Rio de Janeiro - Brasil; Cadernos de Saúde Pública 1:432-446, 1985.

25. Marzochi MCA, Marzochi KBF. Tegumentary and visceral leishmaniasis in Brazil - Emerging anthropozoonosis and possibilities for their control. Cadernos de Saúde Pública 10:359-375. 1994.

26. Menegola IA, Lobo MSC, Pithan OA, Vargá I, Ramos AR. Primeiro relatório do Distrito Sanitário Yanomami: Avaliação das atividades e diagnóstico de saúde, Coordenadoria Regional de Roraima. Fundação Nacional de Saúde, Boa Vista, 1991.

27. Nandy A, Addy M, Guba SK, Chaudrhuri D, Chatterjee P. Co-existent kalaazar and malaria in India. Transactions of the Royal Society of Tropical Medicine and Hygiene 89:516, 1993.

28. Paes MG, Araújo Filho NA, Guerra MVF, Barros MLB, Fé NF, Sá RC, Ramos ED. Ocorrência de Lutzomyia longipalpis ( Lutz \& Neiva, 1912) no Estado de Roraima, Brasil In: Resumos do XI Congresso Brasileiro de Parasitologia, Rio de Janeiro p.65, 1989.

29. Paes MG, Passos LNM, Guerra ALS, Araújo Filho NA, Guerra MVF, Barros MLB, Santos D, Fé NF, Sena CAR, Roseli MP, Lainson R. Isolamento e caracterização de Leishmania de dois casos humanos de calazar do Estado de Roraima. In: Resumos do XXXI Congresso da Sociedade Brasileira de Medicina Tropical, São Paulo p. 238, 1995.

30. Pelegrini M, Magalhães E. Relatório da situação sanitária e dos serviços de atenção a saúde das comunidades Yanomami e Ye'kuana (Maiongong). Distrito Sanitário Yanomami, Coordenadoria Regional de Roraima. Fundação Nacional de Saúde, Boa Vista, 1994.

31. Pondé R, Mangabeira Filho 0, Jansen G. Alguns dados sobre a leishmaniose visceral americana e doença de Chagas no Nordeste Brasileiro. Memórias do Instituto Oswaldo Cruz. 37: 333-362. 1942.

32. Ryan L, Lainson R, Shaw, Habib FNY. Ecologia de flebotomíneos ( Diptera: Psychodidae Phlebotominae) na Região Amazônica. In: Instituto Evandro Chagas ( org) 50 anos de contribuição às ciências biológicas e a medicina tropical. Fundação Serviços de Saúde Pública e Editora Globo, Ministério da Saúde, Belém, 2 vols, 1986.

33. Senra MS, Pimentel PSR, Souza PEFP. Leishmaniose visceral em Santarém - PA: Aspectos gerais do controle, inquerito sorológico em cães e tratamento dos casos humanos. Revista Brasileira de Malariologia e Doenças Tropicais 37:47-59, 1982

34. Sherlock IA. Observações sobre Calazar em Jacobina, Bahia. I - Histórico e dados preliminares. Revista Brasileira de Malariologia e Doenças Tropicais 21:523-534, 1969.

35. Sherlock IA, Almeida SP. Observações sobre Calazar em Jacobina, Bahia. V - Resultados de medidas profiláticas. Revista Brasileira de Malariologia e Doenças Tropicais 175-182. 1970.

36. Sherlock IA, Santos AC. Leishmaniose visceral na zona de Jequié, Estado da Bahia Revista Brasileira de Malariologia e Doenças Tropicais:441-448. 1964.

37. World Health Organization. The Leishmaniasis. Technical Series Informal - 701. Geneva, 1984. 